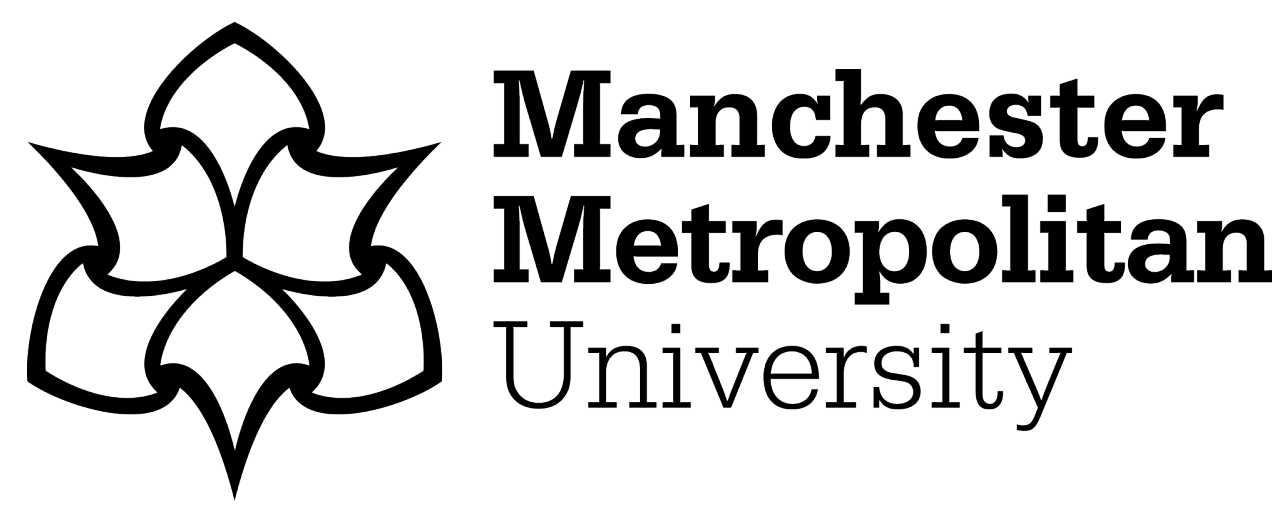

Zhang, X ORCID logoORCID: https://orcid.org/0000-0002-8790-0313, Wang, $Z$ and Liu, $Q$ (2016) Prediction of pressure drop and flow distribution in disc-Type transformer windings in an OD cooling mode. IEEE Transactions on Power Delivery, 32 (4). pp. 1655-1664. ISSN 0885-8977

Downloaded from: https://e-space.mmu.ac.uk/625853/

Version: Accepted Version

Publisher: IEEE

DOI: https://doi.org/10.1109/TPWRD.2016.2557490

Please cite the published version 


\title{
Prediction of Pressure Drop and Flow Distribution in Disc Type Transformer Windings in an OD Cooling Mode
}

\author{
Xiang Zhang, Zhongdong Wang, Member, IEEE, and Qiang Liu, Member, IEEE
}

\begin{abstract}
In this paper, a method for predicting pressure drop and flow distribution in disc-type transformer windings in an oil forced and directed (OD) cooling mode is proposed. First, dimensional analyses are conducted to identify the independent dimensionless variables that affect pressure drop over and flow distribution in the winding. Next, parametric sweeps are performed with computational fluid dynamics (CFD) simulations under isothermal flow conditions. Finally, pressure drop and flow distribution results obtained from the simulations are correlated with the previously identified dimensionless variables to derive correlation equations. These equations have been verified over a range of different isothermal and non-isothermal flow cases and applied to identify the criteria for the occurrence of reverse flow resulting from a combination of pass inlet flow rate and winding geometry. In addition, the method provides an insight into the controlling parameters for determining the minimum oil velocity in horizontal cooling ducts.
\end{abstract}

Index Terms - Dimensional analysis, disc type winding, flow distribution, OD cooling mode, pressure drop, transformer

\section{NOMENCLATURE}

$\bar{c}_{p} \quad$ Mean specific heat of the oil $(J /(\mathrm{kg} \cdot \mathrm{K}))$

$C_{p d} \quad$ Pressure drop coefficient over the winding passes

$H_{\text {disc }} \quad$ Disc vertical (axial) height $(m)$

$H_{\text {duct }} \quad$ Horizontal (radial) duct height $(m)$

$\dot{m} \quad$ Mass flow rate in the pass $(\mathrm{kg} / \mathrm{s})$

$n_{1} \quad$ Number of passes in the winding

$n_{2} \quad$ Number of discs per pass

$N_{d e} \quad$ Number of domain mesh elements

$P_{f i} \quad$ Volumetric flow proportion in horizontal duct $i$

$\Delta P \quad$ Static pressure drop $(\mathrm{Pa})$

$Q \quad$ Total power loss in pass 1 and pass $2(W)$

$r \quad$ Rounding radius of the strand (m)

$R_{i n n} \quad$ Winding inner radius (m)

Re Reynolds number at the pass inlet $\left(\rho \cdot \bar{v}_{\text {in }} \cdot 2 W_{\text {duct }} / \mu\right)$

$\mathrm{T} \quad$ Temperature in Kelvin (K)

$T_{b 1} \quad$ Oil bulk temperature at the inlet of pass $1(K)$

$T_{b 3} \quad$ Oil bulk temperature at the inlet of pass $3(\mathrm{~K})$

$\bar{v}_{i} \quad$ Average oil velocity at the entrance of $\operatorname{duct} \tilde{i}(\mathrm{~m} / \mathrm{s})$

Manuscript received on 2nd September 2015, revised on $18^{\text {th }}$ December 2015 and $22^{\text {nd }}$ February 2016, accepted on $18^{\text {th }}$ April 2016.

X. Zhang, Z.D. Wang and Q. Liu are with the School of Electrical and Electronic Engineering at The University of Manchester, Manchester, M13 9PL, UK (e-mail: zhongdong.wang@ manchester.ac.uk). $\bar{v}_{i n} \quad$ Average pass inlet oil velocity $(\mathrm{m} / \mathrm{s})$

$W_{\text {disc }} \quad$ Disc horizontal (radial) width (m)

$W_{\text {duct }} \quad$ Vertical (axial) duct width (m)

$\alpha \quad$ Dimensionless horizontal duct height $\left(H_{\text {duct }} / W_{\text {duct }}\right)$

$\beta \quad$ Dimensionless disc axial height $\left(H_{\text {disc }} / W_{\text {duct }}\right)$

$\gamma \quad$ Dimensionless disc radial width $\left(W_{\text {disc }} / W_{\text {duct }}\right)$

$\rho \quad$ Oil density $\left(\mathrm{kg} / \mathrm{m}^{3}\right)$

$\mu \quad$ Oil dynamic viscosity $(\mathrm{Pa} \cdot \mathrm{s})$

\section{INTRODUCTION}

$\mathrm{T}$ ransformer life expectancy is generally determined (with the exception of premature failure due to faults) by the temperature of the hottest point in the winding, which is referred to as hot spot [1]. The primary objective of transformer thermal design is to control the hot spot temperature within certain limitations, as specified in standards e.g. IEC 60076-2 and IEEE C57.91 [2, 3].

Heat generation and dissipation in the winding are the critical factors that determine the hot spot temperature. Heat generation in the winding is the result of resistive and eddy current losses. Heat dissipation is usually facilitated by oil circulation between the winding and the cooler/radiator. In disc-type transformer windings, flow distribution in the horizontal (radial) cooling ducts should ideally match the loss distribution [4].

Various thermo-hydraulic network models have been proposed to determine the oil flow and heat transfer processes in the winding [5-7]. The performance of these network models depends crucially on the coefficient correlation equations adopted. These correlation equations are for pressure losses (mainly minor losses) to determine flow distribution in the winding, and for the Nusselt numbers to determine the heat transfer coefficients in the horizontal cooling ducts. They are usually obtained from literature pertaining to circular pipe flow and 2D duct heat transfer processes. The applicability of these traditional correlation equations proved to be unsatisfactory when compared with the performance of newly established correlation equations derived from computational fluid dynamics (CFD) models [810]. It is probably worth mentioning that the correlation equations proposed in [9] were partially verified by measuring the pressure drop over one pass of a test rig; however flow distributions obtained from the calibrated network model were significantly different from those obtained from 2D CFD 
simulations, especially for the cases of high oil flow rates [11].

The correlation equations for minor pressure losses of flow over elbows, in dividing, and merging $\mathrm{T}$-junctions have been greatly improved in [10]. However, the discrepancy between flow distributions in a winding pass from a CFD simulation and a network model with the newly developed minor loss correlation equations is still not negligible (figure 8 in [10]). This could be because flows in the adjoining horizontal ducts interact with one another, whereas a single horizontal duct was employed in [10] in the procedure for deriving the minor pressure drop correlation equations. Therefore, consideration of a whole pass is necessary to capture the complete characteristics of pressure drop and flow distribution in disctype transformer windings.

In addition to CFD calibrations for the correlation equations, experiments were conducted to calibrate the Nusselt numbers in horizontal ducts [12]. Isothermal flow experiments were also conducted to determine the flow distribution and total pressure drop in a disc-type winding [13]. The influence of the number of discs per pass, ratio of horizontal duct height to vertical (axial) duct width and the effect of the elevation of the cooling system were investigated experimentally as well as via network modelling and CFD simulations using a finite difference method [13, 14].

Fluid flow and heat transfer in transformer windings are coupled. This is self-evident for a natural cooling mode (ON) where buoyancy is the only driving force. For ON cooling mode, the temperature profile at the inlet of a pass and the subsequent hot-streak dynamics can significantly affect the flow distribution in the pass $[15,16]$. For oil forced and directed (OD) cooling mode, pressure generated by the pump dominates the flow pattern and therefore the effects of hotstreak dynamics and buoyancy force are much less influential.

In this paper, parameters that affect pressure drop and flow distribution in an OD cooling mode under isothermal flow conditions are identified and grouped into dimensionless groups in section II. The influences of these dimensionless parameters on pressure drop and flow distribution are then quantified by conducting 640 sets of parametric sweeps with CFD simulations in section III. Correlation equations for pressure drop over and flow distribution in the winding with winding geometry and oil flow rate are developed in section IV and verified in section $\mathrm{V}$. The practical implications of these correlation equations are presented in section VI, followed by discussion and conclusion in section VII and VIII.

\section{Dimensional ANALYSES ON PRESSURE DROP AND Flow DISTRIBUTION IN DISC-TYPE TRANSFORMER WINDING PASS}

Any physically significant equation must be dimensionally homogeneous, and this is the premise for dimensional analysis. Dimensional analysis can reduce the number of independent variables and simplify the procedure for analyzing physical problems enormously $[17,18]$.

\section{A. Winding Geometry of Interest}

A disc-type winding possesses an angular periodicity dictated by the number of spacers present along the circumference of the disc. A segment of one pass between two adjoining sets of spacers is shown in Fig. 1(a). Since oil flow in the winding segment is a quasi-2D flow, the winding geometry can be further reduced to an axisymmetric 2D approximation, omitting the details in the vicinity of spacers and strips. The 2D approximation would lead to larger cross section area compared to the real situation with spacers and strips and therefore lower oil velocities for the same oil mass flow rate. This limitation can be partially compensated by applying an area correction coefficient, as indicated in [19]. The 2D geometry of a 3-pass winding with 6 discs per pass is shown in Fig. 1(b), where the details of the cross section of a strand are illustrated in Fig. 1(c).

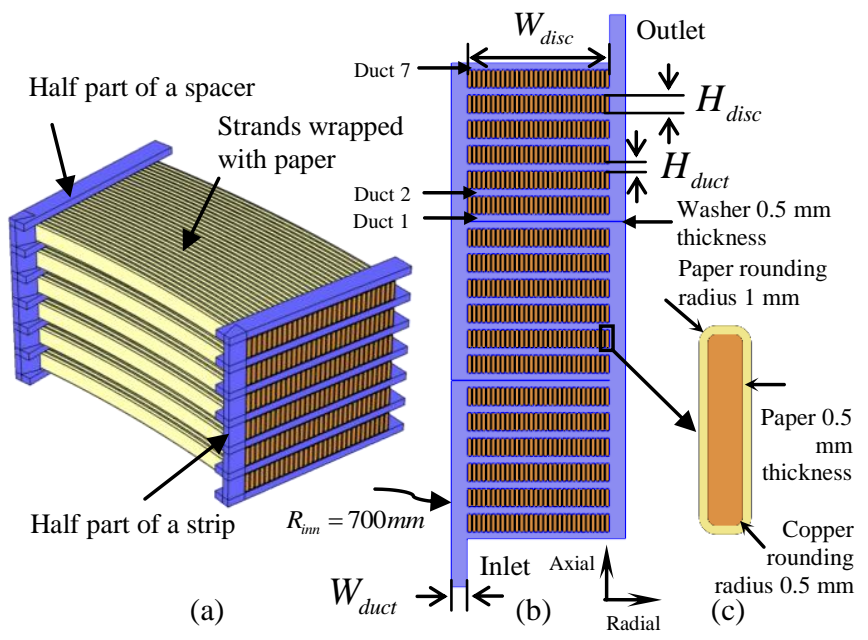

Fig. 1. Disc-type winding geometry. (a) 3D geometry of a segment of one pass between two adjoining sets of spacers. (b) 2D axisymmetric geometry of a 3-pass case. (c) Geometric details of the cross section of a strand. The annotations are added to facilitate dimensional analyses in section II part B and part C. The geometrical dimensions are for baseline cases in section III part B and part D.

\section{B. Dimensional Analysis on Pressure Drop}

The following expression is deemed sufficient to determine the static pressure drop over the winding $(\Delta P)$ :

$\Delta P=f\left(n_{1}, n_{2}, \bar{v}_{i n}, \rho, \mu, H_{\text {duct }}, H_{\text {disc },} W_{\text {disc }}, W_{\text {duct }}, R_{\text {inn }}, r\right)$

Repeating parameters are set to be $\bar{v}_{\text {in }}, \rho$, and $W_{\text {duct }}$, which are combined with the remaining parameters to transform (1) into the dimensionless form:

$\frac{\Delta P}{\rho \bar{v}_{\text {in }}^{2} / 2}=C_{p d}=g\left(n_{1}, n_{2}, \frac{\rho \bar{v}_{\text {in }}\left(2 W_{\text {duct }}\right)}{\mu}, \frac{H_{\text {duct }}}{W_{\text {duct }}}, \frac{H_{\text {disc }}}{W_{\text {duct }}}, \frac{W_{\text {disc }}}{W_{\text {duct }}}, \frac{R_{\text {inn }}}{W_{\text {duct }}}, \frac{r}{W_{\text {duct }}}\right)$

where $\rho \cdot \bar{v}_{i n}^{2} / 2$ is the dynamic pressure at the pass inlet; $2 W_{\text {duct }}$ is the hydraulic diameter of the vertical cooling duct.

\section{Dimensional Analysis on Flow Distribution}

Flow distribution in a pass for inlet at inner vertical duct differs slightly from that of inlet at outer vertical duct. In this analysis we focus on the case of flow inlet at the inner vertical duct, as is the case in the third pass in Fig. 1(b).

The average oil velocity in horizontal cooling duct $i$, which can be any duct in the pass, is expressed in the form of:

$\bar{v}_{i}=f_{i}\left(n_{2}, \bar{v}_{i n}, \rho, \mu, H_{d u c t}, H_{d i s c}, W_{d i s c}, W_{d u c t}, R_{i n n}, r\right)$ 
Similarly, $\bar{v}_{\text {in }}, \rho$, and $W_{\text {duct }}$ are chosen to be repeating parameters, resulting in the dimensionless form of:

$$
\frac{\bar{v}_{i}}{\bar{v}_{i n}}=g_{i}\left(n_{2}, \frac{\rho \bar{v}_{i n} 2 \cdot W_{\text {duct }}}{\mu}, \frac{H_{\text {duct }}}{W_{\text {duct }}}, \frac{H_{\text {disc }}}{W_{\text {duct }}}, \frac{W_{\text {disc }}}{W_{\text {duct }}}, \frac{R_{\text {inn }}}{W_{\text {duct }}}, \frac{r}{W_{\text {duct }}}\right)
$$

As the functional form of $g_{i}$ has not been determined yet, it is permissible to modify these dimensionless groups. The $\bar{v}_{i} / \bar{v}_{i n}$ is replaced by the product of itself and $H_{d u c t} / W_{d u c t}$, which is actually the volumetric flow proportion in duct $i$.

$$
\begin{aligned}
& \frac{\bar{v}_{i}}{\bar{v}_{i n}} \cdot \frac{H_{\text {duct }}}{W_{\text {duct }}}=P_{f i}=g_{i}^{\prime}\left(n_{2}, \frac{\rho \bar{v}_{\text {in }}\left(2 W_{\text {duct }}\right)}{\mu},\right. \\
&\left.\frac{H_{\text {duct }}}{W_{\text {duct }}}, \frac{H_{\text {disc }}}{W_{\text {duct }}}, \frac{W_{\text {disc }}}{W_{\text {duct }}}, \frac{R_{\text {inn }}}{W_{\text {duct }}}, \frac{r}{W_{\text {duct }}}\right)
\end{aligned}
$$

where $\bar{v}_{i} \cdot H_{\text {duct }}$ represents oil flow rate in horizontal duct $i$, $\bar{v}_{i n} \cdot W_{\text {duct }}$ represents oil flow rate at the pass inlet.

\section{Determination of Functional forms}

According to the dimensional analyses, pressure drop coefficient $\left(C_{p d}\right)$ and flow proportion in duct $i\left(P_{f i}\right)$ can be determined by (2) and (5), respectively. However, the functional forms of (2) and (5) are not specified by the analyses themselves, but need to be obtained either by experimentation or theoretical calculations.

In this paper, CFD simulations are conducted. The results for $C_{p d}$ and $P_{f i}$ are extracted from the simulations and then correlated with the dimensionless parameters via curve fitting to obtain the functional forms.

\section{PARAMETRIC SWEEPS}

Before performing parametric sweeps, a set of test cases were conducted to check the validity of the foregoing dimensional analyses and to provide some insight into the effects of the dimensionless groups.

\section{A. CFD Simulations}

COMSOL Multiphysics is employed to implement the theoretical calculations for which the Navier-stokes equations and the continuity equation are solved directly using a finite element method. Because the Reynolds number (Re) at the pass inlet is smaller than the criterion value for transition to turbulence, no turbulence model is included.

In all CFD simulations, the meshes consist of layers of thin rectangles for the flow boundary layers and triangles for the main flow domains. Mesh refinement studies are conducted to guarantee mesh-independent solutions. It is found that the number of rectangle layers has negligible influence on the results of flow distribution and pressure drop. Therefore, 2 layers of thin rectangles are used in all the meshes. For a typical CFD case, such as the benchmark case to be shown in section III part B, the mesh consists of 1,159,948 domain elements and 48,048 boundary elements. The number of degrees of freedom is $2,143,368$. The meshing time is 24 minutes and the calculation time is 9 minutes. The results of mesh refinement study of the benchmark case are shown in Table I. The tracking parameters at each mesh size or number of domain elements $\left(N_{d e}\right)$ are pressure drop over the three passes $(\Delta P)$ and the average oil velocity in the bottom duct of pass $3\left(\bar{v}_{1}\right)$. According to the varying trend of the tracking parameters with the number of domain elements shown in Table I, the mesh strategy corresponding to mesh 4 is adopted for all the CFD simulations.

TABLE I

Mesh REFINEMENT STUdy RESUlts OF THE BENCHMARK CASE

\begin{tabular}{c|ccccc}
\hline \hline Strategy & mesh 1 & mesh 2 & mesh 3 & mesh 4 & mesh 5 \\
\hline$N_{d e}$ & 390,862 & 568,428 & 966,554 & $1,159,948$ & $2,866,688$ \\
\hline$\Delta P(\mathrm{~Pa})$ & 445.42 & 473.85 & 493.46 & 495.19 & 495.54 \\
\hline $\bar{v}_{1}(\mathrm{~mm} / \mathrm{s})$ & 46.760 & 46.539 & 46.672 & 46.607 & 46.617 \\
\hline \hline
\end{tabular}

\section{B. Computational Tests of Dimensional Analyses}

The geometries of the test cases resemble those of $\mathrm{HV}$ windings for transmission transformers. In the tests, $n_{1}$ is fixed to be $3, n_{2} 6, R_{i n n} 700 \mathrm{~mm}, r 1 \mathrm{~mm}$, washer thickness $0.5 \mathrm{~mm}$. The radial width of the strand is fixed to be $3 \mathrm{~mm}$, whereas the axial height of the strand is adjustable. A mineral oil is used as the coolant whose temperature (in Kelvin) dependent density and viscosity are

$\rho=-0.65110 \times T+1067.0$

$\mu=5.9622 \times 10^{-5} \times \exp \left(\frac{707.64}{T-165.20}\right)$

Under these test conditions, we presume that $C_{p d}$ and $P_{f i}$ are dominated by $\operatorname{Re}$ at the pass inlet, dimensionless horizontal duct height $(\alpha)$, dimensionless disc axial height $(\beta)$, and dimensionless disc radial width $(\gamma)$.

1) Test cases

Five test cases are performed with $\operatorname{Re}, \alpha, \beta$, and $\gamma$ being kept identical in all the cases. Case 1 is set as a benchmark where the vertical duct width $\left(W_{\text {duct }}\right)$ is $10 \mathrm{~mm}$, horizontal duct height $\left(H_{\text {duct }}\right) 4 \mathrm{~mm}(\alpha=0.4)$, disc axial height $\left(H_{\text {disc }}\right) 12 \mathrm{~mm}$ $(\beta=1.2)$, disc radial width $\left(W_{\text {disc }}\right) 90 \mathrm{~mm}(\gamma=9)$, the average pass inlet velocity $0.3 \mathrm{~m} / \mathrm{s}$, oil density $876 \mathrm{~kg} / \mathrm{m}^{3}$ and dynamic viscosity $0.01506 \mathrm{~Pa} \cdot \mathrm{s}$, being the values at $293.15 \mathrm{~K}\left(20{ }^{\circ} \mathrm{C}\right)$ based on (6) and (7), respectively. Therefore, $\mathrm{Re}$ at the pass inlet is calculated as equal to 349. Case 2 is the same as case 1 , except that pass inlet velocity is changed to $0.5 \mathrm{~m} / \mathrm{s}$, and $\mu$ is changed accordingly to keep Re remaining constant at 349 . Similarly, in case 3 the pass inlet velocity is changed to 0.4 $\mathrm{m} / \mathrm{s}$, whereas $\rho$ is changed to keep Re identical. In case 4 , $W_{\text {duct }}$ is changed to $8 \mathrm{~mm}$ and the other parameters are changed accordingly to maintain the constant values of $\operatorname{Re}, \alpha, \beta$ and $\gamma$. Case 5 is the same as case 4 except that $W_{\text {duct }}$ is changed to $12 \mathrm{~mm}$.

2) Comparison of Results

The results of the $C_{p d}$ of each case for 1 pass, 2, and 3 passes are shown in Table II. The differences in $C_{p d}$ ' $s$ among the five cases for the same number of passes are negligible, as shown by each row. In addition, $C_{p d}$ rise approximately linearly with the number of passes, as shown by each column. 
Average oil velocity of each horizontal duct was extracted at the same radial position. The volumetric oil flow proportion in one duct was then calculated by dividing its average velocity by the sum of all the average velocities in that pass.

The results of $P_{f i}$ in the second and third passes of each case are compared in Fig. 2. As can be seen, flow distributions for the same pass are similar to one another in all cases with negligible differences. In addition, there are only slight differences in flow distributions between pass 2 and pass 3 , for which the flow directions are opposite.

The consistency of these test results with the forgoing dimensional analyses indicates that the analyses in section II are reliable. It is worth noticing that in the test cases $R_{i m n}$ and $r$ are fixed, whereas $W_{\text {duct }}$ for case $1(10 \mathrm{~mm})$, case $4(8 \mathrm{~mm})$ and case $5(12 \mathrm{~mm})$ are different. Therefore, $R_{\text {inn }} / W_{\text {duct }}$ and $r / W_{\text {duct }}$ are different with the ratio of their maximum value to the minimum value being 1.5. However, the results of $C_{p d}$ and $P_{f i}$ in these cases are so close that we conclude that these two dimensionless parameters are negligible in the investigated range when compared with $\operatorname{Re}, \alpha, \beta$, and $\gamma$.

From these test results, we conclude that it is reasonable to confine the study to investigate the influence of $\operatorname{Re}, \alpha, \beta$, and $\gamma$ on $C_{p d}$ for 3 passes and $P_{f i}$ in the third pass. The number of discs per pass is fixed to be 6 . The investigated winding geometry is shown schematically in Fig. 1(b). The values of the investigated parameters, apart from $\operatorname{Re}, \alpha, \beta$, and $\gamma$, are the same as those specified in the test cases and shown in Fig. 1(b) and Fig. 1(c).

TABLE II

PRESSURE DRop COEFFICIENTS OF THE FIVE TEST CASES

\begin{tabular}{c|ccccc}
\hline \hline Pass No. & Case 1 & Case 2 & Case 3 & Case 4 & Case 5 \\
\hline Pass 3 & 3.8218 & 3.8259 & 3.8226 & 3.8693 & 3.7712 \\
\hline Pass 2, 3 & 8.0770 & 8.0852 & 8.0786 & 8.0947 & 8.0454 \\
\hline Pass 1, 2, 3 & 11.643 & 11.656 & 11.646 & 11.712 & 11.557 \\
\hline \hline
\end{tabular}

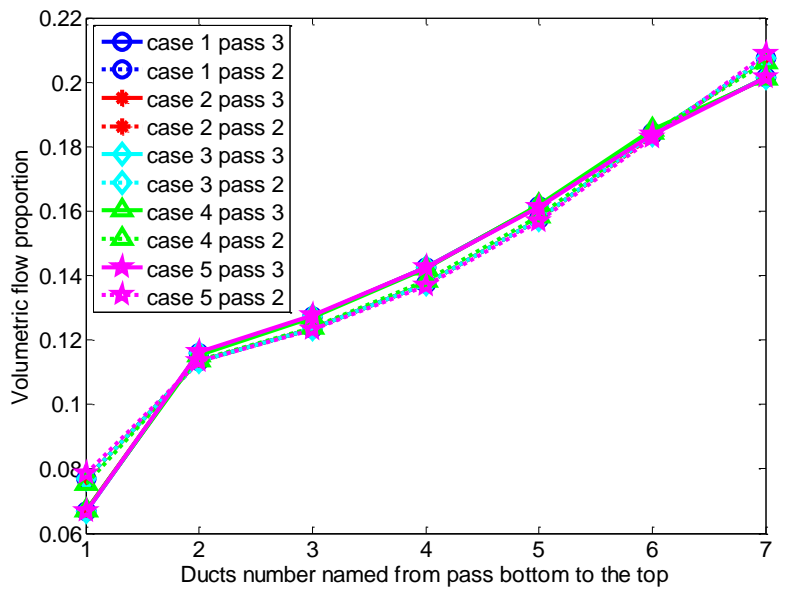

Fig. 2. Flow distribution in pass 2 and pass 3 of the five test cases.

\section{Ranges of Parameters of Interest}

The ranges of geometric parameters of interest taken from [20] are shown in Table III. For the OD cooling mode, Re at the pass inlet is set to range from 50 to 1200 , as shown in table
IV. The ranges of the dimensionless geometric parameters $(\alpha, \beta, \gamma)$ are determined based on the data range in Table III, and are selected to keep the resultant winding geometry practical.

\section{CFD Simulation Strategies}

The parametric sweep of Re is relatively easy as when the winding geometry is fixed, mesh only needs to be done once. The required Re can be accommodated by adjusting fluid properties. This is permissible, as is indicated by the test cases. However, to accommodate all of the 80 combinations of $\alpha$, $\beta$, and $\gamma$, which represent 80 winding geometries, each geometry would need a separate CFD model and meshing process.

It is chosen to relate the vertical duct width to $\beta$ as illustrated in Table IV, where the vertical duct width in $\mathrm{mm}$ is the denominator of each $\beta$ value. For example, when $\beta=10 / 10$, the vertical duct width is set to be $10 \mathrm{~mm}$, and the actual winding geometries can be determined by combining all the different $\alpha$ 's and $\gamma^{\prime} s$, as demonstrated in Table V.

TABLE III

RANGES OF SELECTED GEOMETRIC PARAMETERs [20]

\begin{tabular}{|c|c|c|c|}
\hline$W_{d u c t}(\mathrm{~mm})$ & $H_{d u c t}(\mathrm{~mm})$ & $H_{\text {disc }}(\mathrm{mm})$ & $W_{d i s c}(\mathrm{~mm})$ \\
\hline $6-12$ & $3-6$ & $7-20$ & $40-170$ \\
\hline \multicolumn{4}{|c|}{ TABLE IV } \\
\hline $\operatorname{Re}$ & & \multicolumn{2}{|c|}{$50,100,200,400,600,800,1000,1200$} \\
\hline$\alpha$ & & \multicolumn{2}{|c|}{$\begin{array}{lllll}0.25, & 0.3, & 0.4, & 0.5, & 0.6\end{array}$} \\
\hline$\beta$ & & \multicolumn{2}{|c|}{$8 / 12, \quad 10 / 10, \quad 16 / 8, \quad 18 / 6$} \\
\hline$\gamma$ & & \multicolumn{2}{|c|}{$6,9,12,15$} \\
\hline
\end{tabular}

TABLE V

\begin{tabular}{cccc}
\multicolumn{4}{c}{ ACtUAL Winding Dimensions For $\beta=10 / 10\left(W_{d u c t}, H_{d u c t}, H_{d i s c}, \mathrm{~W}_{\text {disc }}\right)$} \\
\hline \hline$(10,2.5,10,60)$ & $(10,2.5,10,90)$ & $(10,2.5,10,120)$ & $(10,2.5,10,150)$ \\
\hline$(10,3.0,10,60)$ & $(10,3.0,10,90)$ & $(10,3.0,10,120)$ & $(10,3.0,10,150)$ \\
\hline$(10,4.0,10,60)$ & $(10,4.0,10,90)$ & $(10,4.0,10,120)$ & $(10,4.0,10,150)$ \\
\hline$(10,5.0,10,60)$ & $(10,5.0,10,90)$ & $(10,5.0,10,120)$ & $(10,5.0,10,150)$ \\
\hline$(10,6.0,10,60)$ & $(10,6.0,10,90)$ & $(10,6.0,10,120)$ & $(10,6.0,10,150)$ \\
\hline \hline
\end{tabular}

All the geometrical dimensions are in $\mathrm{mm}$.

In this table, $\alpha$ varies with row number ranging from 0.25 to $0.6 ; \gamma$ varies with column number ranging from 6 to 15 .

\section{Correlation Equations}

In total, 640 CFD simulations are conducted for the parametric sweeps, from which $C_{p d}$ for three passes and $P_{f i}$ of the third pass are computed based on the simulation results. $C_{p d}$ and $P_{f i}$ are then correlated with $\operatorname{Re}, \alpha, \beta$, and $\gamma$.

\section{A. Correlation equations for pressure drop coefficient}

The results of $C_{p d}$ against Re can be plotted on a log-log scale. The relationship for several exemplary geometries are illustrated in Fig. 3. The other geometries lead to similar varying trend. The $C_{p d}$ 's for a particular geometry fall on a curve that is straight at the beginning and then levels off at the 
end, indicating a power law as well as an exponentially decreasing dependence of the $C_{p d}$ on the increasing Re. This varying trend is similar to that of the drag coefficient of a smooth sphere immersed in a uniform stream [18].

The fitting method of correlating $C_{p d}$ to $\operatorname{Re}, \alpha, \beta$, and $\gamma$ closely follows the strategy adopted in [10]. The functional forms are

$$
\left\{\begin{array}{l}
C_{p d}=a_{1} \frac{1000}{\operatorname{Re}} e^{a_{2} \mathrm{Re} / 1000} \\
a_{i}=b_{i 1}(4 \alpha)^{b_{i 2}} e^{4 \alpha b_{i 3} 3} ; i=\{1,2\} \\
b_{i j}=c_{i j 1} \beta^{c_{i j 2}} e^{c_{i j 3} \beta} ; j=\{1,2,3\} \\
c_{i j k}=d_{i j k 1} \gamma^{3}+d_{i j k 2} \gamma^{2}+d_{i j k 3} \gamma+d_{i j k 4} ; k=\{1,2,3\}
\end{array}\right.
$$

where $a_{i}, b_{i j}, c_{i j k}$ are dummy parameters, and 72 parameter $d$ 's are generated.

In each fitting layer, least-squares fittings are adopted. The final fitting results are $R^{2}=0.99994$, the maximum absolute error 1.4998 , and the maximum relative error $17.115 \%$.

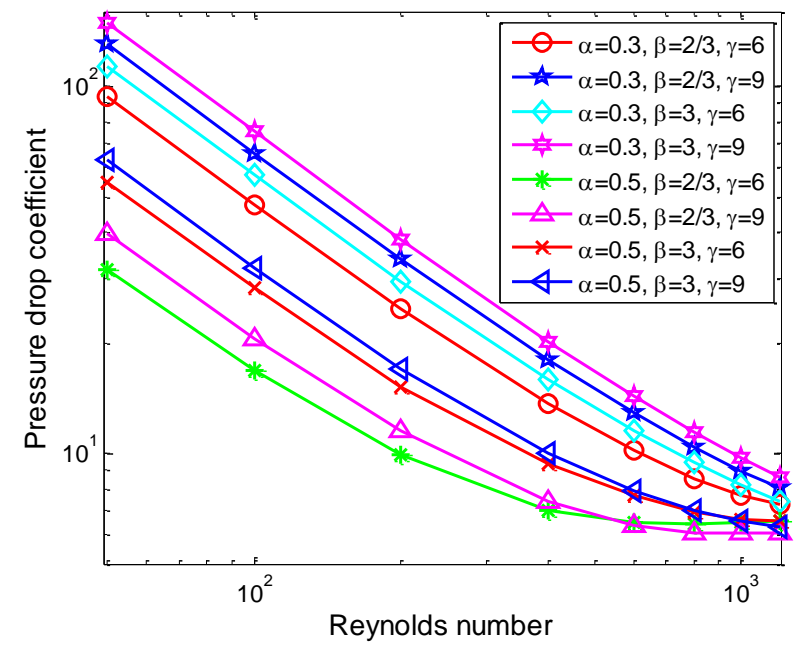

Fig. 3. Varying trends of pressure drop coefficients against the Reynolds numbers for several exemplary geometries.

\section{B. Correlation equations for flow distribution}

The same fitting strategy is adopted to correlate $P_{f i}$ in the third pass to Re, $\alpha, \beta$, and $\gamma$. The consistency of the correlation equations is tested subsequently.

1) Correlation equations of the flow proportions

The functional forms of the correlations are:

$$
\left\{\begin{array}{l}
P_{f i}=\frac{a_{i 1}}{\operatorname{Re}^{1 / 3}}+a_{i 2} \ln \operatorname{Re}+a_{i 3} \operatorname{Re}^{1 / 3}+a_{i 4} ; i=\{1,2,3,4,5,6,7\} \\
a_{i j}=b_{i j 1} \alpha^{3}+\mathrm{b}_{i j 2} \alpha^{2}+\mathrm{b}_{i j 3} \alpha+\mathrm{b}_{i j 4} ; \quad j=\{1,2,3,4\} \\
b_{i j k}=c_{i j k 1} \beta^{3}+\mathrm{c}_{i j k 2} \beta^{2}+\mathrm{c}_{i j k 3} \beta+\mathrm{c}_{i j k 4} ; \quad k=\{1,2,3,4\} \\
c_{i j k m}=d_{i j k m 1} \gamma^{3}+d_{i j k m 2} \gamma^{2}+d_{i j k m 3} \gamma+d_{i j k m 4} ; m=\{1,2,3,4\}
\end{array}\right.
$$

where $a_{i j}, b_{i j k}, \quad c_{i j k m}$ are dummy parameters, and 256 parameter $d$ 's are generated for each $P_{f i}$.
Similarly, least-squares fittings are adopted for each fitting layer. The fitting results for each duct of the third pass are shown in Table VI.

The reason why maximum relative errors are huge for duct 1-4 compared with those for duct 5-7 is that reverse flow or stagnant flow, which results in extremely small flow proportion values at the bottom ducts, occurs under certain conditions in the investigated parameter range.

2) Consistency Test of correlation equations

Flow proportion in each duct is correlated independently. The consistency of these correlation equations means that the sum of all the horizontal duct flow proportions in the third pass should be approximately 1 , i.e.

$\sum_{i=1}^{7} P_{f i} \approx 1$

This consistency requirement should be obeyed by the 640 simulated data points as well as by the points in their vicinity. The ranges of the parameters for the consistency test are shown in Table VII. The consistency has been tested outside the range for which the formulae have been set up. In total, 43,200 cases have been tested based on the correlation equations. The minimum sum of all duct flow proportions in the third pass is 0.99983, and the maximum is 1.0001, indicating an excellent consistency of the correlation equation sets.

TABLE VI

FITTING RESULTS FOR EACH DUCT IN THIRD PASS

\begin{tabular}{c|ccc}
\hline \hline Duct number & $R^{2}$ & $\begin{array}{c}\text { Maximum } \\
\text { absolute error }\end{array}$ & $\begin{array}{c}\text { Maximum relative } \\
\text { error (\%) }\end{array}$ \\
\hline 1 & 0.99852 & 0.0081035 & 263.16 \\
2 & 0.99112 & 0.020949 & 1497.4 \\
3 & 0.98843 & 0.024869 & 354.62 \\
4 & 0.97816 & 0.021017 & 1223.5 \\
5 & 0.99780 & 0.0052281 & 3.0054 \\
6 & 0.99952 & 0.0073791 & 2.9525 \\
7 & 0.99946 & 0.010038 & 2.9733 \\
\hline \hline
\end{tabular}

TABLE VII

RANGES OF TESTED PARAMETERS

\begin{tabular}{c|cccc}
\hline \hline Parameter & Minimum & Maximum & Interval & $\begin{array}{c}\text { No. of } \\
\text { values }\end{array}$ \\
\hline $\operatorname{Re}$ & 50 & 1600 & 50 & 32 \\
$\alpha$ & 0.1 & 0.8 & 0.05 & 15 \\
$\beta$ & 0.6 & 3.0 & 0.3 & 9 \\
$\gamma$ & 6 & 15 & 1 & 10 \\
\hline \hline
\end{tabular}

\section{VERIFICATIONS AND PREDICTIONS}

The aim of performing dimensional analyses, conducting parametric sweeps, and correlating the results of $C_{p d}$ and $P_{f i}$ to the dimensionless parameters is to use the correlation equations to predict pressure drops and flow distributions in various OD cooling conditions. To validate the correlating strategies adopted in section IV, comparing the results from the correlation equations and the CFD simulations for new cases must be conducted. Because the correlation equations are derived from isothermal flow conditions, its suitability to 
be applied for prediction under non-isothermal flow conditions is also demonstrated in this section.

\section{A. Predictions of isothermal flow cases}

The new winding geometry for the isothermal flow prediction cases is the same as those in the parametric sweeps, except that $W_{\text {duct }}=7 \mathrm{~mm}, H_{\text {duct }}=3 \mathrm{~mm}(\alpha=3 / 7), H_{\text {disc }}=12 \mathrm{~mm}$ $(\beta=12 / 7)$, and $W_{\text {disc }}=90 \mathrm{~mm}(\gamma=90 / 7)$. The oil temperature is set to be $40{ }^{\circ} \mathrm{C}$. The first pass inlet velocity is set to range from $0.1 \mathrm{~m} / \mathrm{s}$ to $0.5 \mathrm{~m} / \mathrm{s}$ with an interval of $0.1 \mathrm{~m} / \mathrm{s}$, resulting in corresponding Reynolds number of 170.14 , 340.28, 510.42, 680.56, and 850.70.

The correlation equations from section IV are used directly to obtain $C_{p d}$ for three passes and $P_{f i}$ in the third pass. These pressure drops and flow distributions are also obtained from new CFD simulations for comparison purpose.

1) Comparisons of pressure drops for isothermal flows

The pressure drops obtained from the correlation equation set and the new CFD simulations are shown in Fig. 4. Compared with the CFD results, the maximum absolute and relative errors of the correlation equation set are approximately $17 \mathrm{~Pa}$ and $4.5 \%$, respectively.

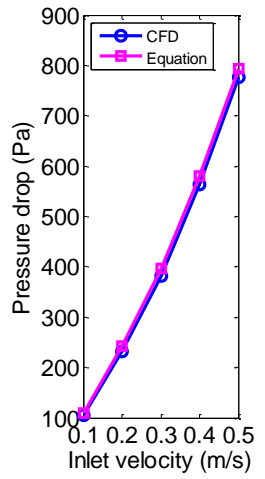

(a)

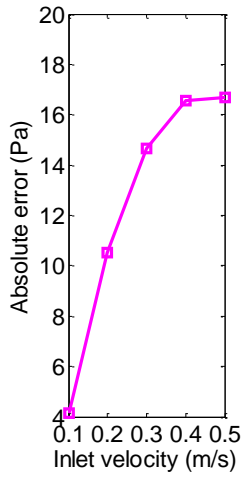

(b)

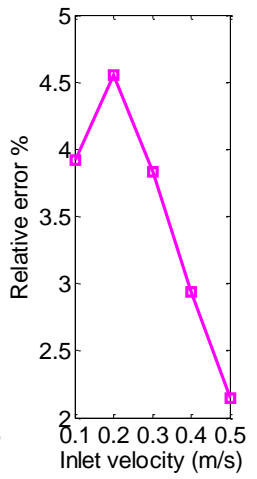

(c)
Fig. 4. Pressure drop under isothermal flow conditions. (a) Comparison of pressure drops from the correlation equation set and the CFD simulations. (b) Absolute errors. (c) Relative errors.

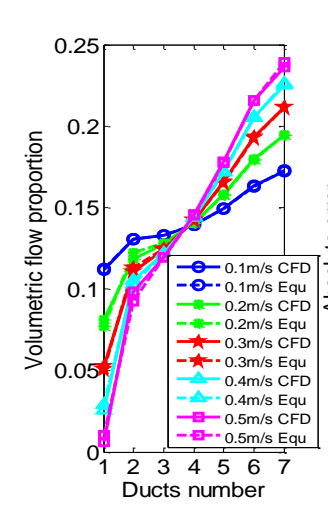

(a)

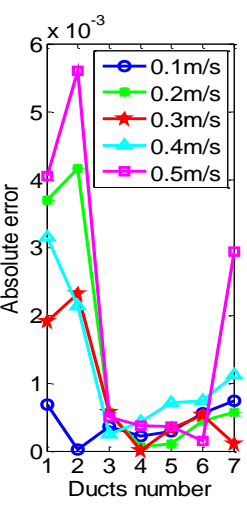

(b)

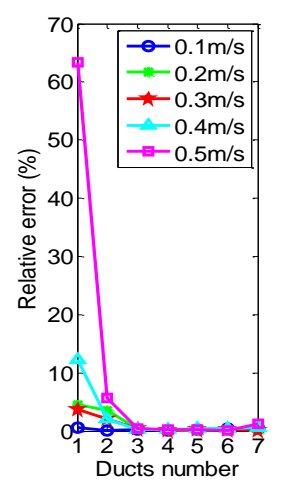

(c)
Fig. 5. Flow distribution under isothermal flow conditions for different pass inlet velocities with horizontal ducts numbered from the pass bottom to the top. (a) Comparison of flow distributions obtained from correlation equations and CFD simulations. (b) Absolute errors. (c) Relative errors.

\section{2) Comparisons of flow distribution for isothermal flows}

Flow distributions obtained from the correlation equations and the new CFD simulations are shown in Fig. 5. Results from these two methods are close to each other with the maximum absolute error being smaller than $0.6 \%$ of the total volumetric flow rate. The large relative errors for duct 1 at the pass inlet velocities of $0.5 \mathrm{~m} / \mathrm{s}$ and $0.4 \mathrm{~m} / \mathrm{s}$ are because of the small flow proportions in these two cases. The relative errors for the other cases are smaller than $5 \%$.

\section{B. Predictions of non-isothermal flow cases}

The winding geometry and flow conditions for nonisothermal flow cases are the same as those for the isothermal flow cases. Since the hot-spot temperature is not the purpose of the tests, a uniform loss distribution is imposed to all winding discs. An average heat flux of $1248 \mathrm{~W} / \mathrm{m}^{2}$ is prescribed to the copper surface of each strand, as shown in Fig. 1(c), which corresponds to an average heat flux of 3505.8 $W / \mathrm{m}^{2}$ on the surface of each disc. The oil inlet temperature of the first pass is set to be $40{ }^{\circ} C$. Conjugate Heat Transfer (CHT) simulations are performed which couple the fluid flow and heat transfer processes completely without adopting Boussinesq approximation to obtain the pressure drops and flow distributions. These CHT results are set as benchmarks to be compared with prediction results from the correlation equations.

For non-isothermal flow, oil bulk temperature at the inlet of the third pass is different from that of the first pass and therefore needs to be calculated to modify the Reynolds number. Energy conservation is adopted to calculate the oil bulk temperature at the inlet of the third pass:

$T_{b 3}=T_{b 1}+\frac{Q}{\dot{m} \bar{c}_{p}}$

Iterative procedure is needed so as to determine the mean specific heat at the mean temperature of $T_{b 1}$ and $T_{b 3}$.

1) Comparisons of pressure drops for non-isothermal flows

The pressure drops over the three passes obtained from the correlation equation set with modified Reynolds numbers and those from the CHT simulations are shown in Fig. 6. The discrepancies in the pressure drops are quite similar to those in Fig. 5, indicating that the effects of non-isothermal flow have negligible influence on the pressure drop over the winding pass.

2) Comparisons of flow distribution for non-isothermal flows

The flow distributions obtained from the correlation equations with modified Reynolds numbers and the CHT simulations are shown in Fig. 7. Compared with Fig. 5, the absolute and relative errors are larger, especially in the top duct (duct 7) and for the case of the winding pass inlet velocity being $0.1 \mathrm{~m} / \mathrm{s}$ which corresponds to an average horizontal duct velocity of $0.033 \mathrm{~m} / \mathrm{s}$. These discrepancies are due to the impact of hot-streak dynamics and buoyancy force, which are more influential in the case of a lower flow rate. However, for a higher flow rate it is less influential, as in the cases of pass inlet velocities larger than $0.1 \mathrm{~m} / \mathrm{s}$ where the maximum absolute error of flow proportion in each duct is smaller than approximately $1 \%$ of the total volumetric flow rate. The huge relative errors for duct 1 at pass inlet velocities 
being $0.5 \mathrm{~m} / \mathrm{s}$ and $0.4 \mathrm{~m} / \mathrm{s}$ are also because of the small flow proportions in these two cases. The relative errors for the other cases are within $15 \%$.

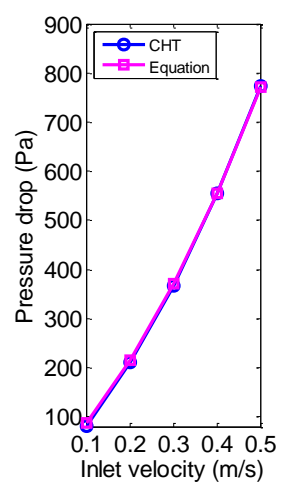

(a)

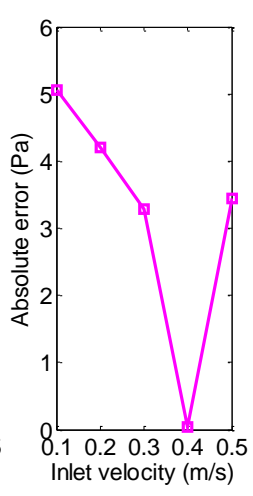

(b) (c)

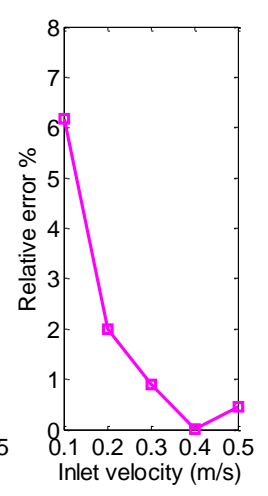

Fig. 6. Pressure drop under non-isothermal flow condition. (a) Comparison of pressure drop over three passes from CHT simulations and the correlation equation set. (b) Absolute errors. (c) Relative errors.

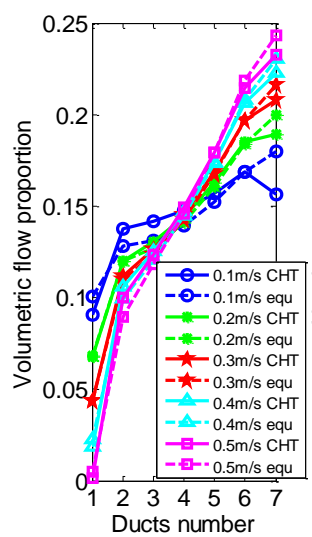

(a)

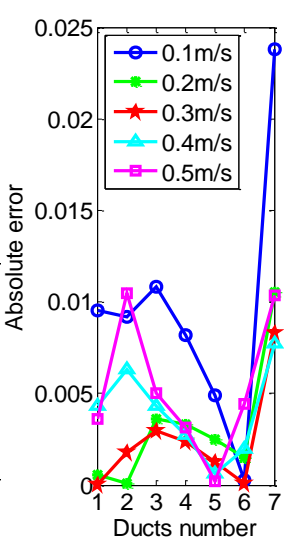

(b)

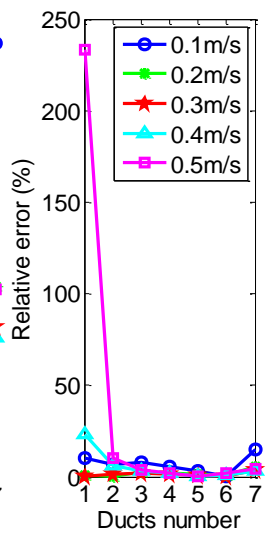

(c)
Fig. 7. Flow distribution under non-isothermal flow condition for different pass inlet velocities with ducts numbered from the pass bottom to the top. (a) Comparison of flow distributions from CHT simulations and correlation equations. (b) Absolute errors. (c) Relative errors.

\section{PRACTICAL IMPLICATIONS}

By using these correlation equations, the pressure drop and flow distribution of a disc-type transformer winding in an OD cooling mode can be easily investigated.

\section{A. Predicting the occurrence of reverse flow in a pass}

Reverse flow can occur in the winding in an OD cooling mode, as indicated by the experimental work in [13] and the computational work in [21]. Once reverse flow occurs it can jeopardize the cooling performance because the reverse flow usually leads to a low flow rate and some horizontal cooling ducts may even suffer from stagnant flows.

Since the reverse flow happens initially in the bottom duct of a pass, the correlation equation for flow proportion in duct 1 can be applied to investigate the criterion for the occurrence of reverse flow in the winding.

Previous study demonstrated that flow proportion in a duct is a function of $\operatorname{Re}$ at the pass inlet and the geometric parameters ( $\alpha, \beta$, and $\gamma$ ), more importantly, it is mainly controlled by $\operatorname{Re}$ and the ratio of horizontal duct height to vertical duct width $(\alpha)$. To show the variation of the flow proportion in duct 1 with different combinations of $\operatorname{Re}$ and $\alpha$, $\beta$ is fixed to be 1 and $\gamma$ to be 10 .

The variation of flow proportion in duct 1 with Re and $\alpha$ is shown in Fig. 8. There are two distinct regions in Fig. 8, where region 1 is a reversal-free region and region 2 would experience reverse flow. It can be seen that flow proportion in duct 1 decreases monotonically with increasing $\operatorname{Re}$ and $\alpha$.

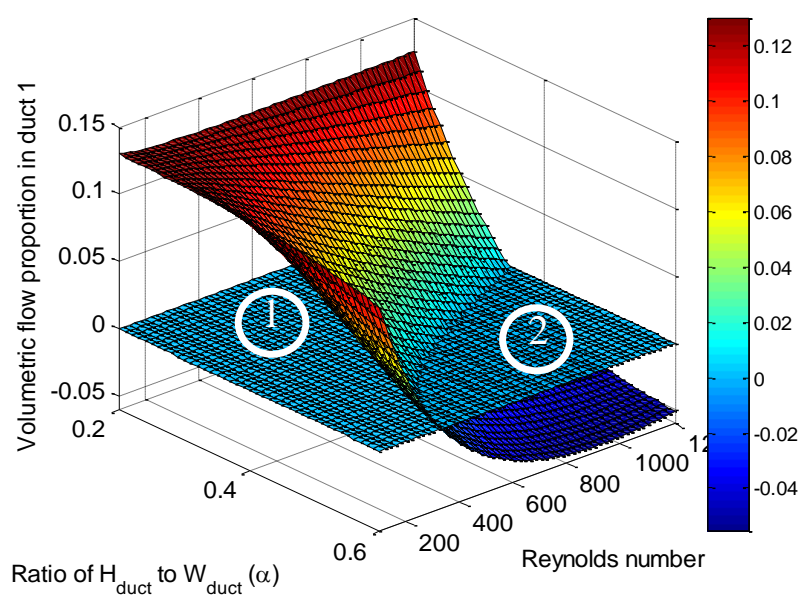

Fig. 8. Volumetric flow proportion in duct 1 for different combinations of $\mathrm{Re}$ and $\alpha$. Two regions are identified with region 1 being reversal-free and region 2 experiencing reverse flow.

\section{B. Improving the cooling performance}

Flow distribution in the winding pass changes with the pass inlet flow rate, and a higher flow rate does not necessarily result in a better cooling performance. In fact, a too high flow rate can result in reverse flow in the pass bottom duct, as illustrated in Fig. 8.

In an OD cooling mode, flow proportion rises with duct number in a pass, numbered from the pass bottom to the top, as can be seen in Fig. 5 and Fig. 7. For a relative uniform loss distribution, the overall cooling performance is therefore determined by the velocity in the bottom horizontal duct, which is dictated by both the pass inlet flow rate and flow proportion in the bottom duct. Such an observation helps to systematically adjust and optimize the combination of design parameters including winding geometric parameters and pass inlet flow rate.

A demonstration case is given here, where $H_{\text {duct }}$ is fixed at $4 \mathrm{~mm}$, the oil temperature at $40^{\circ} \mathrm{C}$, and the other parameters being the same as those in section VI part A. The average oil velocity at the entrance of duct 1 is:

$\bar{v}_{1}=\frac{\bar{v}_{i n} P_{f 1}}{\alpha}$

In order to obtain $P_{f i}$, Re is determined from:

$\operatorname{Re}=\frac{\bar{v}_{\text {in }} \rho\left(2 W_{\text {duct }}\right)}{\mu}=\frac{\bar{v}_{\text {in }} \rho\left(2 \mathrm{H}_{\text {duct }}\right)}{\mu \alpha}$

where $\rho$ and $\mu$ are calculated from (6) and (7), respectively.

Finally, the variation of $\bar{v}_{1}$ with $\alpha$ and $\bar{v}_{i n}$, as shown in Fig. 9 , is obtained by applying the correlation equation for $P_{f i}$. As 
can be seen, the maximum value of average oil velocity in duct $1\left(\bar{v}_{1}\right)$ is not achieved at the maximum average pass inlet velocity $\left(\bar{v}_{i n}\right)$. For example, when the ratio of horizontal duct height to vertical duct width is $0.25(\alpha=0.25)$, the maximum $\bar{v}_{1}$ is achieved at $\bar{v}_{\text {in }} \approx 0.3 \mathrm{~m} / \mathrm{s}$. In addition, when $\alpha$ increases, $\bar{v}_{\text {in }}$ needs to decrease to achieve the corresponding maximum $\bar{v}_{1}$. For example, when $\alpha=0.6$, the maximum $\bar{v}_{1}$ is achieved at $\bar{v}_{\text {in }}=0.1 \mathrm{~m} / \mathrm{s}$ which is the lowest pass inlet velocity in the study.

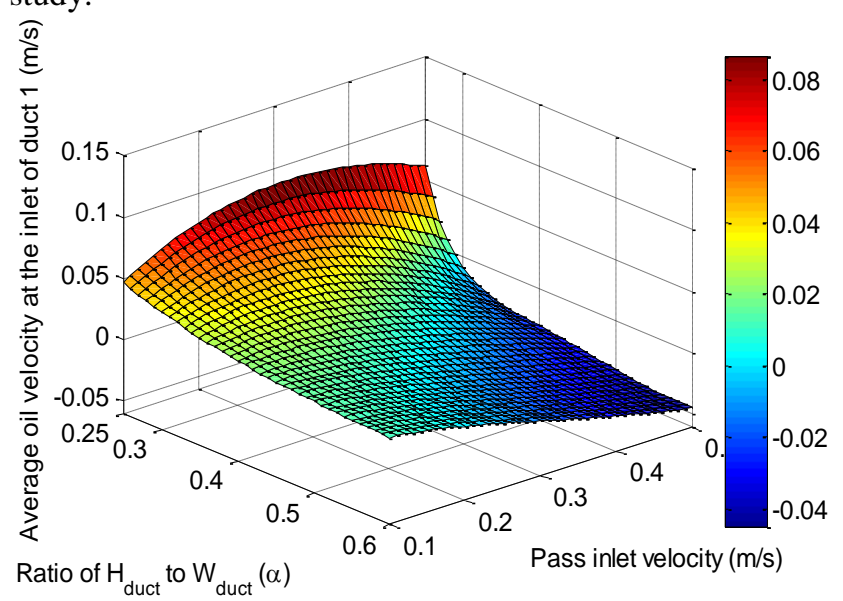

Fig. 9. Variation of average velocity in bottom horizontal duct with $\alpha$ and pass inlet velocity.

\section{DISCUSSION}

Coolant flow distribution in a disc-type transformer winding has a direct impact on the temperature distribution in the winding. Pressure drop over the winding is also an important parameter for selecting pumps and determining the split of coolant flow among different windings connected hydraulically in parallel. Flow distribution in and pressure drop over the winding can be modelled by using network models or CFD simulations. However, even with the modelling solutions, one could still be baffled as to how flow distribution and pressure drop are controlled by the variables of winding geometries, fluid properties and coolant flow rate because of the large number of the possible combinations of all these variables.

Dimensional analysis, which has been widely adopted in fluid mechanics, provides a method of simplifying the relationship among the variables without losing accuracy. After performing dimensional analysis, we may find that variables that seem to be unrelated are grouped into a dimensionless parameter and different combinations of these variables can possibly lead to identical effect in a dimensionless sense. In addition, the decrease of the number of variables by transforming them into dimensionless groups makes parametric sweeps much more manageable. CFD simulations have the advantage of accuracy over network models, though they require much higher computational effort. With the help of CFD simulations, parametric sweeps of the identified dimensionless parameters are performed. The dimensionless CFD results are then correlated with the identified dimensionless parameters to form predictive correlation equations.
Results in section V demonstrate that the correlation equations are capable of predicting pressure drop and flow distribution for both isothermal flow and non-isothermal flow conditions in an OD cooling mode with acceptable accuracy. This is because firstly and fundamentally the pressure drop over and flow distribution in the winding do not change their varying trends drastically when the dimensionless parameters are altered, and secondly the correlation equations can preserve the accuracy of the CFD simulations as long as the parametric sweeps have appropriate intervals within the selected ranges of the controlling dimensionless parameters and the curve fitting procedures are sufficiently accurate.

By applying the correlation equations, we are able to identify the criteria for the occurrence of reverse flow and the conditions of achieving the maximum value of the lowest duct oil velocity in the pass.

It is worth emphasizing that the method presented in this paper can be considered to be not constrained to the number of passes of the winding. The number of passes in the winding does not affect flow distribution within a pass for isothermal flow conditions. Even opposite flow directions would just lead to negligible effect on flow distribution, as shown in Fig. 2. For non-isothermal flow conditions, which are the practical situations, the effect of buoyancy force, hot-streak dynamics and the increase of oil temperature with the elevation along the winding would affect the flow distribution. The effect of temperature increase can be accounted for by modifying the Reynolds number at the pass inlet, as shown in (11). By modifying the Reynolds number reasonable flow distribution approximation for the non-isothermal flow condition can be obtained, as shown in Fig. 7. Pressure drop over the winding can be reasonably considered as proportional to the number of passes, as shown in Table II. Isothermal flow and nonisothermal flow have almost the same pressure drop characteristics, as shown by Fig. 4 and Fig. 6. Therefore, a 3pass winding would be representative for the simulations with pass 2 and pass 3 showing the effect of opposite flow directions and pass 1 acting as a buffer zone to eliminate the effect of prescribing velocity profile at the inlet of pass 1 .

In addition, these correlation equations for pressure drop and flow distribution may be used to replace the hydraulic part of network model to improve the performance of network modelling. In order to cover all the possible disc-type winding geometries, additional parametric sweeps and correlations need to be performed for the cases of:

1. Different numbers of discs per pass.

2. Different inner and outer vertical duct width. This would generate another dimensionless parameter of the ratio of outer vertical duct width to inner vertical duct width.

3. Non-uniform horizontal duct heights which follow a certain pattern or rule, for example, the case of alternately varied horizontal duct height.

4. Different schemes of positioning washers and the case of zigzag winding created with seals next to the discs, etc.

Eventually, a library that can accommodate all the cases could be established.

Furthermore, this method is performed on a pass-by-pass basis. Therefore, the results of pressure drop and flow distribution would be applicable to windings consisting of passes with different numbers of discs, provided that the 
generalization of the method for new cases presented above has been accomplished.

\section{CONCLUSION}

Dimensional analyses were conducted to identify the independent dimensionless variables that affect the pressure drop over disc-type transformer windings and the oil flow distribution in the windings in an OD cooling mode.

Parametric sweeps for a 3-pass winding with 6 discs per pass were performed, using CFD simulations under isothermal flow conditions. The pressure drop coefficient $\left(C_{p d}\right)$ for the three passes and flow proportion in duct $i\left(P_{f i}\right)$ for the third pass were extracted from the simulations and correlated to the dimensionless parameters to form predictive correlation equations. The correlation equations were then verified over a range of different isothermal and non-isothermal flow cases.

The correlation equation for flow proportion in the pass bottom duct was applied to identify the criteria for the occurrence of reverse flow, an important phenomenon in an OD cooling mode, resulting from a combination of pass inlet flow rate and winding geometry, and resulting in lower oil flow rate and therefore higher hot-spot temperature than expected. In addition, the method provides an insight into the controlling parameters for determining the minimum oil velocity in horizontal cooling ducts. These two applications indicate that higher pass inlet flow rate does not necessarily mean better cooling performance and an appropriate combination of winding geometry and pass inlet flow rate can optimize the cooling performance.

\section{ACKNOWLEDGMENT}

The authors would like to express their gratitude to M\&I Materials, National Grid, Scottish Power, Shell, TJ|H2b Analytical Services, UK Power Networks and Weidmann Electrical Technology for their financial and technical contributions to the Transformer Research Consortium at The University of Manchester.

\section{REFERENCES}

[1] M. Heathcote, $J \&$ P transformer book (page 158), Thirteenth ed.: Newnes, 2007.

[2] Temperature rise for liquid-immersed transformers, IEC standard 60076-2, 2011.

[3] IEEE Guide for Loading Mineral-Oil-Immersed Transformers and Step-Voltage Regulators, IEEE standard C57.91, 2011.

[4] J. Zhang, and X. Li, "Oil cooling for disk-type transformer windings-Part II: Parametric studies of design parameters," IEEE Trans. Power Del., vol. 21, no. 3, pp. 1326-1332, 2006.

[5] A. J. Oliver, "Estimation of transformer winding temperatures and coolant flows using a general network method," Proc. Inst. Elect. Eng., vol. 127, pp. 395-405, 1980.

[6] Z. R. Radakovic, and M. S. Sorgic, "Basics of detailed thermalhydraulic model for thermal design of oil power transformers," IEEE Trans. Power Del., vol. 25, no. 2, pp. 790-802, 2010.

[7] J. Zhang, and X. Li, "Oil cooling for disk-type transformer windings-part 1: theory and model development," IEEE Trans. Power Del., vol. 21, no. 3, pp. 1318-1325, 2006.

[8] W. Wu, Z.D. Wang, A. Revell, P. Jarman, "Computational fluid dynamics calibration for network modelling of transformer cooling oil flows-part I heat transfer in oil ducts," IET Electric Power Applications, vol. 6, no. 1, pp. 19-27, 2012.

[9] W. Wu, Z.D. Wang, A. Revell, P. Jarman, "Computational fluid dynamics calibration for network modelling of transformer cooling
flows-Part II: pressure loss at junction nodes," IET electric power applications, vol. 6, no. 1, pp. 28-34, 2012.

[10] J. Coddé, W. Van der Veken, and M. Baelmans, "Assessment of a hydraulic network model for zig-zag cooled power transformer windings," Appl. Therm. Eng., vol. 80, pp. 220-228, 2015.

[11] A. Weinläder, W. Wu, S. Tenbohlen, Z.D. Wang, "Prediction of the oil flow distribution in oil-immersed transformer windings by network modelling and computational fluid dynamics," IET electric power applications, vol. 6, no. 2, pp. 82-90, 2012.

[12] J. Zhang, X. Li, and M. Vance, "Experiments and modeling of heat transfer in oil transformer winding with zigzag cooling ducts," Appl. Therm. Eng., vol. 28, no. 1, pp. 36-48, 2008.

[13] M. Nakadate, K. Toda, K. Sato et al., "Gas cooling performance in disc winding of large-capacity gas-insulated transformer," IEEE Trans. Power Del., vol. 11, no. 2, pp. 903-908, 1996.

[14] E. Rahimpour, M. Barati, M. Schafer, "An investigation of parameters affecting the temperature rise in windings with zigzag cooling flow path", Appl. Therm. Eng., vol. 27, no. 11-12, pp. 1923-1930, 2007.

[15] F. Torriano, M. Chaaban, and P. Picher, "Numerical study of parameters affecting the temperature distribution in a disc-type transformer winding," Appl. Therm. Eng., vol. 30, no. 14, pp. 2034-2044, 2010.

[16] A. Skillen, A. Revell, H. Iacovides et al., "Numerical prediction of local hot-spot phenomena in transformer windings," Applied Thermal Engineering, vol. 36, pp. 96-105, 2012.

[17] Ain A. Sonin. (2001) Dimensional Analysis (2nd ed. page 34-49,) [Online] :http://web.mit.edu/2.25/www/pdf/DA_unified.pdf

[18] R. W. Fox, A. T. McDonald, and P. J. Pritchard, Fluid Mechanics, (page 290-301 and 296), New York: John Wiley \& Sons, 2012.

[19] F. Torriano, P. Picher, M. Chaaban, "Numerical investigation of 3D flow and thermal effects in a disc-type transformer winding", Appl. Therm. Eng., vol. 40, pp. 121-131, 2012.

[20] J. Zhang, X. Li, "Coolant flow distribution and pressure loss in ONAN transformer windings. Part II: Optimization of design parameters," IEEE Trans. Power Del., vol. 19, pp. 194-199, 2004.

[21] A. Weinläder, and S. Tenbohlen, "Thermal-hydraulic investigation of transformer windings by CFD-modelling and measurements." Paper D-22, Proc.16th ISH, South Africa, 2009.

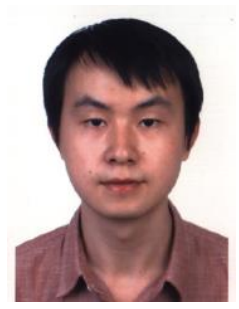

Xiang Zhang received the B.Eng. degree in Electrical and Electronic Engineering (2012) from Xi'an Jiaotong University in China. Since 2014, he started his Ph.D. programme at the Electrical Energy and Power Systems Group of the School of Electrical and Electronic Engineering at The University of Manchester in the UK. His research programme is on transformer thermal modelling.

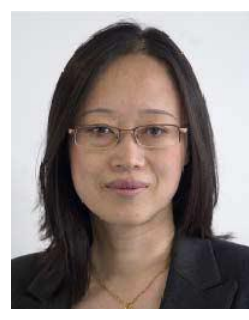

Zhongdong Wang received the BSc and the MSc degrees in high voltage engineering from Tsinghua University in 1991 and 1993 and the $\mathrm{PhD}$ degree in electrical engineering from UMIST in 1999. Currently she is a Professor of High Voltage Engineering of the School of Electrical and Electronic Engineering at The University of Manchester. Her research interests focus on power transformers covering all aspects of modelling and simulation, materials \& systems, and asset management policies.

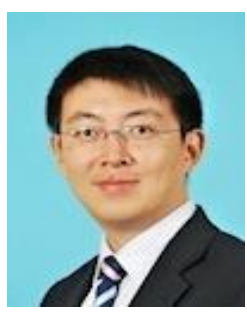

Qiang Liu (S'08-M'12) obtained the B.Eng. degree in electrical engineering (2005) and the M.Eng. degree in high voltage and electrical insulation (2008) from Xi'an Jiaotong University in China, and the $\mathrm{Ph} . \mathrm{D}$. degree in electrical power engineering (2011) from The University of Manchester in the UK. Currently he is a Lecturer of the School of Electrical and Electronic Engineering at The University of Manchester. His research interests are on prebreakdown and breakdown phenomena in liquids, ester transformer liquids, streaming electrification, ageing of insulating materials, transformer asset management and high voltage testing. 